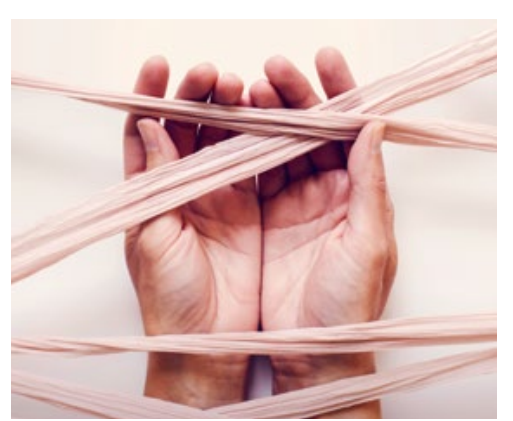

\section{CPD}

Julie Gordon, Graeme C Miller, Helena Britt

\section{Background}

There is currently no accepted standard definition for chronic conditions.

\section{Objective}

The objective of this article is to provide an overview of the characteristics that define health conditions as chronic.

\section{Discussion}

A list of health conditions classified as chronic should be made available to all general practitioners and policymakers in Australia. This will assist in the claims process for chronic disease management and aid the selection of patients for healthcare home enrolment.

Based on assessment of the usual duration, prognosis, sequelae and pattern of recurrence or deterioration, a defined list of chronic conditions classified according to the International Classification of Primary Care, version 2 (ICPC-2) is available for consideration.

\title{
What are chronic conditions that contribute to multimorbidity?
}

THE STUDY of multimorbidity has escalated exponentially across the world, as patient-centred care grows in importance. Recent research suggests that $26 \%$ of the Australian population has two or more chronic conditions, and $15.8 \%$ has three or more. ${ }^{1}$ There is contention about how many chronic conditions (ie two or more, three or more) should constitute 'multimorbidity'1 but more importantly, there is no accepted standard definition for what constitutes 'chronic' conditions.

The concept of using chronic conditions as a measure of need or complexity is also recognised by the Australian Consortium for Classification Development in the development of the International Statistical Classification of Diseases and Related Health Problems, 10th revision, Australian Modification (ICD-10-AM), the classification used in Australian hospitals. ${ }^{2}$ It is also used by the federal government in the Chronic Disease Management (CDM) items of the Medicare Benefits Schedule (MBS) for the management of patients with 'chronic and complex care needs'. Assessment of patient eligibility for the CDM program 'is essentially a matter for the GP to determine, using their clinical judgement and taking into account both the eligibility criterion and the general guidance'. ${ }^{3}$ However, little guidance is given to general practitioners (GPs) to assist them in this assessment.

The definition of a chronic condition put forward by the Australian government, is 'one that has been, or is likely to be, present for six months or more'. There is no list of eligible conditions, but focus is often given to the common chronic conditions (eg asthma, cancer, cardiovascular disease, diabetes, musculoskeletal conditions, stroke). ${ }^{3}$ This is a very simplistic definition. Duration of a condition is only one of many factors that need to be considered, and the examples are limited.

In 2004, we recognised that a standard definition of 'chronic' was needed for research into the population prevalence and general practice management of chronic conditions. We reviewed the available literature to identify the characteristics that define a chronic condition and assessed each of the codes in the International Classification of Primary Care, version 2 (ICPC-2) ${ }^{4}$ against these criteria to develop a list of ICPC-2 codes that represent chronic conditions. ${ }^{5}$ After an extensive literature review, we found multiple possible factors to consider, and identified the criteria most relevant to the definition of chronic conditions. These include the following.

\section{- Expected duration of at least} six months

The length of time required for a condition to be regarded as chronic varies widely in the literature. Some definitions include definite time frames, such as three, six or 12 months, while others use terms such as 'prolonged'. We concluded that duration alone is inadequate in determining whether a medical condition is chronic.

\section{- Poor prognosis}

The literature strongly indicates that chronic conditions either cannot be 
cured, or there is uncertainty around the possibility of a cure. This underlines the need for regular management by GPs and other members of the patient's healthcare team. ${ }^{5}$

\section{- Consequences or sequelae that affect quality of life}

These consequences may be in the form of complications, limitations of activity or an increased need for medical care.

\section{- Pattern of recurrence or deterioration}

Chronic conditions may have patterns of recurrence and remission, but there may be considerable variance to such a pattern between and within conditions. ${ }^{5}$

When we applied these criteria to ICPC-2, we identified 127 ICPC-2 rubrics (concept labels) as 'chronic'. A further 20 rubrics contained both chronic and non-chronic conditions; for example, 'rag-bag' rubrics, such as code 'K99: Other circulatory diseases', include some chronic and some non-chronic conditions not classifiable elsewhere in ICPC-2. In these 20 mixed rubrics, we further defined the chronicity of each individual condition at the terminology level (ICPC-2 PLUS). ${ }^{6}$ Duration was the criterion most frequently satisfied (98.4\% of chronic rubrics), while $88.2 \%$ of rubrics met at least three of the four criteria. ${ }^{5} \mathrm{~A}$ full list of the conditions classified as chronic according to ICPC-2 is available at http://sydney. edu.au/medicine/fmrc/classifications/ DefiningChronicConditions.pdf. The chronic problems managed most frequently in Australian general practice (based on this list) can be found in Table 7.6 in General practice activity in Australia 2015-16. ${ }^{7}$ Problems commonly managed in general practice that did not satisfy the criteria include immunisations/ vaccinations, urinary tract infections and pregnancy. ${ }^{5}$

In 2015-16, Gordon reviewed the published list of chronic ICPC-2 codes and found that no changes were required - the list remains applicable today. This is not an exhaustive list of all chronic conditions.
Clinical judgement is vital when assessing whether a condition is chronic in an individual patient's clinical care. We suggest that GPs could use this list to broaden their ideas about what constitutes a chronic condition (beyond the most common), especially when considering whether their patients are eligible for management through CDM items.

Multimorbidity cannot be quantified without defining chronic conditions. A standardised list of chronic conditions would be invaluable to multimorbidity researchers, particularly those analysing large patient databases or electronic health record data. For many researchers, the ICPC-2 chronic condition list has filled this gap in Australia (and more widely internationally, 211 citations). The idea of linking chronic conditions to GP payment structures is gaining popularity with the advent of the healthcare homes trial. ${ }^{8}$ If such programs are to succeed, a standardised definition of chronic conditions may aid decisions around patient eligibility for inclusion.

The continued uncertainty regarding what conditions should be defined as chronic needs to be resolved. Clinicians, researchers and administrators need to reach a consensus on exactly what should be included or excluded from a list of chronic conditions using our internationally recognised list as a starting point.

\section{Authors}

Julie Gordon BAppSc (HIM) (Hons), PhD, Research Fellow, National Centre for Classification in Health, Faculty of Health Sciences, University of Sydney, NSW. julie.gordon@sydney.edu.au

Graeme C Miller MBBS, PhD, FRACGP, Honorary Associate Professor, Sydney School of Public Health, Faculty of Medicine, University of Sydney, NSW Helena Britt BA, PhD, Professor of Primary Care Research, Sydney School of Public Health, Faculty of Medicine, University of Sydney, NSW

Competing interests: None.

Provenance and peer review: Commissioned, externally peer reviewed.

\section{References}

1. Harrison C, Henderson J, Miller G, Britt H. The prevalence of diagnosed chronic conditions and multimorbidity in Australia: A method for estimating population prevalence from general practice patient encounter data. PLoS One 2017;12(3):e0172935.

2. Australian Consortium for Classification Development. Overview of supplementary codes for chronic conditions. Sydney: ACCD, 2015. Available at www.accd.net.au/Education. aspx?page=2 [Accessed 26 October 2017].

3. Department of Health. Chronic Disease Management (formerly Enhanced Primary Care or EPC) - GP services. Canberra: DoH, 2017. Available at www.health.gov.au/internet/ main/publishing.nsf/content/mbsprimarycarechronicdiseasemanagement [Accessed 18 August 2017].

4. Classification Committee of the World Organization of Family Doctors. ICPC-2: International Classification of Primary Care. 2nd edn. Oxford: Oxford University Press, 1998.

5. O'Halloran J, Miller GC, Britt H. Defining chronic conditions for primary care with ICPC-2. Fam Pract 2004;21(4):381-86.

6. University of Sydney. ICPC-2 PLUS: The BEACH coding system NSW: University of Sydney, 2017. Available at http://sydney.edu.au/health-sciences/ ncch/icpc-2-plus/overview.shtml [Accessed 18 August 2017].

7. Britt H, Miller GC, Henderson J, et al. General practice activity in Australia 2015-16. Sydney: Sydney University Press, 2016. Available at purl.library.usyd.edu.au/sup/9781743325131 [Accessed 16 November 2017].

8. Department of Health. Health Care Homes. Canberra: DoH, 2016. Available at www.health. gov.au/internet/main/publishing.nsf/Content/ AD51EBE397452EF5CA2580F700164BAD/ \$File/ Health\%20Care\%20Homes\%20info\%20 book.pdf [Accessed 18 August 2017]. 